\title{
Article \\ Flurbiprofen: A Study of the Behavior of the Scalemate by Chromatography, Sublimation, and NMR
}

\author{
Magdalena Kwiatkowska ${ }^{1}$, Alicja Wzorek ${ }^{1, *}$, Anna Kolbus ${ }^{1}$ (D), Mariusz Urbaniak ${ }^{1}$, Jianlin Han ${ }^{2}{ }^{(1)}$, \\ Vadim A. Soloshonok ${ }^{3,4}$ (D) and Karel D. Klika ${ }^{5, *}$ \\ 1 Institute of Chemistry, Jan Kochanowski University in Kielce, Uniwersytecka 7, 25-406 Kielce, Poland; \\ magdalena.kwiatkowska@ujk.edu.pl (M.K.); anna.kolbus@ujk.edu.pl (A.K.); \\ mariusz.urbaniak@ujk.edu.pl (M.U.) \\ 2 College of Chemical Engineering, Nanjing Forestry University, Nanjing 210037, China; hanjl@njfu.edu.cn \\ 3 Department of Organic Chemistry I, Faculty of Chemistry, University of the Basque Country UPV/EHU, \\ Paseo Manuel Lardizábal 3, E-20018 San Sebastián, Spain; vadym.soloshonok@ehu.eus \\ 4 IKERBASQUE, Basque Foundation for Science, Alameda Urquijo 36-5, Plaza Bizkaia, E-48011 Bilbao, Spain \\ 5 Molecular Structure Analysis, German Cancer Research Center (DKFZ), Im Neuenheimer Feld 280, \\ D-69120 Heidelberg, Germany \\ * Correspondence: awzorek@ujk.edu.pl (A.W.); k.klika@dkfz.de (K.D.K.); Tel.: +48-41-349-7019 (A.W.); \\ +49-6221-42-4515 (K.D.K.)
}

Citation: Kwiatkowska, M.; Wzorek, A.; Kolbus, A.; Urbaniak, M.; Han, J.; Soloshonok, V.A.; Klika, K.D.

Flurbiprofen: A Study of the Behavior of the Scalemate by Chromatography, Sublimation, and NMR. Symmetry 2021, 13, 543. https://doi.org/ 10.3390/sym 13040543

Academic Editor: Enrico Bodo

Received: 11 March 2021

Accepted: 22 March 2021

Published: 26 March 2021

Publisher's Note: MDPI stays neutral with regard to jurisdictional claims in published maps and institutional affiliations.

Copyright: (c) 2021 by the authors. Licensee MDPI, Basel, Switzerland. This article is an open access article distributed under the terms and conditions of the Creative Commons Attribution (CC BY) license (https:// creativecommons.org/licenses/by/ $4.0 /)$.

\begin{abstract}
Fluoro-4-biphenyl) propionic acid (flurbiprofen), from the phenylalkanoic acid family of nonsteroidal anti-inflammatory drugs (NSAID's), is currently on the pharmaceutical market as a racemate. This racemic compound was tested for its propensity to undergo the self-disproportionation of enantiomers (SDE) phenomenon by various forms of chromatography (SDEvC), such as routine gravity-driven column chromatography, medium-pressure liquid chromatography (MPLC), preparative thin-layer chromatography (PTLC), and size-exclusion chromatography (SEC), as well as by sublimation (SDEvS). Furthermore, examination by nuclear magnetic resonance (NMR) in various solvents found that flurbiprofen exhibited the phenomenon of self-induced diastereomeric anisochronism (SIDA). By measurement of the diffusion coefficient $(D)$, the longitudinal relaxation time $\left(T_{1}\right)$, and the transverse relaxation time $\left(T_{2}\right)$ using NMR, as well as by electrospray ionization-mass spectrometry (ESI-MS) examinations, the preferred intermolecular association was found to be solvent dependent, e.g., heterochiral association was preferred in toluene, while homochiral association was preferred in more polar solvents. This study also attempted, unsuccessfully, to correlate the NMR measurements of flurbiprofen with chromatographic outcomes for the rationalization and prediction of chromatographic results based on NMR measurements. Because the intermolecular hydrogen bonding of the acid groups in flurbiprofen overwhelmingly predominates over other intermolecular interactions, flurbiprofen seemed to represent a good test case for this idea. The behavior of scalemic samples of flurbiprofen is important, as, although it is currently dispensed as a racemate, clinical applications of the $R$ enantiomer have been investigated. SDEvC and SDEvS both have ramifications for the preparation, handling, and storage of enantioenriched flurbiprofen, and this concern applies to other chiral drugs as well.
\end{abstract}

Keywords: flurbiprofen; fluorine-containing pharmaceuticals; self-induced diastereomeric anisochronism (SIDA); enantiomeric analysis; molecular association; NMR; diffusion; molecular chirality; self-disproportionation of enantiomers (SDE)

\section{Introduction}

Studies of both the nuclear magnetic resonance (NMR) phenomenon of self-induced diastereomeric anisotropy (SIDA) and the phenomenon of the self-disproportionation of enantiomers (SDE) have steadily progressed in recent years. SIDA has been described in detail in the ensuing references but briefly: SIDA occurs when chiral molecules that 
associate in solution in a dynamic equilibrium that is fast on the NMR timescale have significant condition-dependent NMR chemical shifts ( $\left.\delta^{\prime} \mathrm{s}\right)$. In such systems, molecules can be present either as single molecules (SM), homochiral associates (HOM), or heterochiral associates (HET) in solution. Because the formation constant for the association of homochiral molecules $\left(K_{\text {hom }}\right)$, i.e., $R$ with $R$ or $S$ with $S$, is likely to be different to the formation constant for the association of heterochiral molecules $\left(K_{\text {het }}\right)$, the positions of the two equilibria will likely be different. Therefore, the observed $\delta$ of a nucleus is the population-weighted average of the $\delta^{\prime}$ s of the nucleus in the three states SM, HOM, and HET [1-3]. Furthermore, as the equilibrium shifts with a change in conditions (e.g., concentration or temperature), the contributions of the $\delta^{\prime}$ s from the SM, HOM, and HET states to the population-weighted average $\delta$ alter accordingly. Enantiopure and racemic solutions can exhibit distinct spectra because of these dynamic effects, and even distinct signals for the two enantiomers can result in the case of scalemates $[1,3,4]$. The SIDA phenomenon with many fine examples in the literature [2-18] has been well reviewed in the past [1,19] and theoretically explained $[3,20]$ along with its potential applications and problems.

It is of interest to determine the solution-state association preference, i.e., whether HOM or HET are favored, and there are a number possible means to do this by NMR, including:

(1) Direct measurements: diffusion coefficients $\left(D^{\prime} s\right)$, longitudinal relaxation times $\left(T_{1} s\right)$, and transverse relaxation times $\left(T_{2} s\right)[2,3]$, although the differences can sometimes be quite small for these measurements and some idea of associate structure may be required if mixed results are obtained for the $T_{1} \mathrm{~s}$ and $T_{2} \mathrm{~s}$.

(2) Variation in sample conditions: plots of enantiomeric titration [2,5], serial dilution, temperature variation, and titration of cofactors (e.g., secondary solvent, acid/base, ionic strength) though some idea of associate structure may be required and care is required in the interpretation of the plots together with appreciation of the $\delta^{\prime} \mathrm{s}$ [21].

(3) Other complex approaches: the interpretation of $\delta^{\prime}$ s and intermolecular nuclear Overhauser effects (NOE's) [3], although intimate knowledge of the structure is likely to be required for $\delta^{\prime}$ s, together with an appreciation of the consequences of varying relative concentrations [21]. Some general structural comprehension at least is also likely to be required in the case of intermolecular NOE's, and suitable spins are not always available for such an analysis.

Each method has its pros and cons, and some methods are broadly applicable, while others are restricted. The variation in sample conditions methods is based on alterations that disrupt or alter the intermolecular interactions giving rise to molecular association, which are subsequently monitored by NMR. It is worth noting that the preference for homoor heterochiral associates is very much state dependent, and there is not a general sense for a compound to always favor one or the other associate under all circumstances.

Knowledge of the solution-state association preference can potentially have practical application: for example, which portion-the racemic portion or the enantiomeric excess (ee) portion-is likely to elute first under chromatographic conditions that will lead to the SDE, a related phenomenon [22-31] also based on the association of chiral molecules [32]. The SDE occurs when a scalemate is subjected to any physicochemical process [33], not just chromatography, and the sample is fractionated, which results in variation of the ee across the fractions. Knowledge of the elution order is particularly applicable for size-exclusion chromatography (SEC) [34]. The intermolecular interactions at the root cause for both the SDE and SIDA phenomena may develop through orbital-orbital interactions, termed autosolvation [35].

In this study, we examined the SDE of 2-(2-fluoro-4-biphenyl)propionic acid (flurbiprofen, Figure 1), from the phenylalkanoic acid family of nonsteroidal anti-inflammatory drugs (NSAID's), by several physicochemical processes to clearly demonstrate the danger that the SDE can pose to drug manufacture and storage if due care is not taken regarding the SDE. Flurbiprofen was selected as an example case since it contained fluorine-a substituent likely to confer enhanced volatility and categorized as an SDE-phoric group [36-39]. When present in a compound, an SDE-phoric group generally enhances the expression of the SDE, 
although the opposite can also happen and the SDE is diminished when it occurs strongly in a compound upon the inclusion of fluorine. While there are numerous examples of fluorine-containing compounds highly expressing the SDE due to the presence of fluorine under a variety of physicochemical processes $[26,34,37,40-50]$, the present work is the first comprehensive SDE study of a fluorinated drug currently present on the pharmaceutical market [51-54]. Thus, scalemic samples of flurbiprofen were tested for their propensity to undergo the SDE phenomenon by various forms of chromatography (SDEvC) and sublimation (SDEvS). Flurbiprofen was also examined by NMR in various solvents to check whether it exhibited the phenomenon of SIDA. NMR measurements, viz. $D, T_{1}$, and $T_{2}$, as well as electrospray ionization-mass spectrometry (ESI-MS), were also applied to determine the solution-state association preference. The principle aim of this study was to correlate NMR measurements with chromatographic outcomes to enable the rationalization and prediction of chromatographic outcomes based on NMR measurements. The strategy was to look for association and strong SIDA effects in the NMR and, hence, identify suitable eluent systems for chromatography. Although it can be very difficult to predict the elution order, the excess enantiomer or the racemic portion, since the intermolecular hydrogen bonding of the acid groups in flurbiprofen should overwhelmingly predominate over other intermolecular analyte-analyte or analyte-stationary phase interactions, flurbiprofen was considered to represent a good test case for this idea. The enantioenriched flurbiprofen that was used in this work was prepared from racemic material using both kinetic (enzymatic reaction) and thermodynamic (diastereomeric salt crystallization) control.<smiles>CC(C(=O)O)c1ccc(-c2ccccc2)c(F)c1</smiles>

Figure 1. Structure of flurbiprofen. The chiral center is marked with "*".

The behavior of scalemic samples of flurbiprofen, a racemic compound [55], is important, as, although it is currently dispensed as a racemate when used as an NSAID, clinical applications of the $R$ enantiomer have been investigated, including as a treatment for Alzheimer's disease in a Phase III clinical trial. The potential dangers that are posed by the SDE during the preparation, handling, and storage of the enantioenriched drug due to the SDE are of concern — and this extends to other chiral drugs as well-since the SDE is ubiquitous and ever-present and, thus, the potential for unintended detrimental effects must never be ignored or underestimated.

\section{Results and Discussion}

\subsection{Resolution of (rac)-Flurbiprofen}

Zhang et al. have reported [56] that the enzymatic enantioresolution of (rac)-flurbiprofen using Candida antarctica lipase provides (S)-flurbiprofen with $91 \%$ ee after $84 \mathrm{~h}$ in tandem with a $67 \%$ conversion of the substrate to ( $R$ )-flurbiprofen methyl ester. In this study, after two days the ee of unconverted flurbiprofen was $86 \%$, dropping to $77 \%$ after three days in tandem with a conversion of $66 \%$.

In order to further improve the ee, (scl)-flurbiprofen was also subjected to diastereomeric salt crystallization $[57,58]$ using either 1-cyclohexyl ethylamine or 1-phenyl ethylamine as the base and ethanol as the solvent for crystallization (Figure 2). For example, the ee of $(S)$-flurbiprofen was increased from $77 \%$ to $95 \%$ by this mean. The Supplementary Materials tabulate various runs (Table S1). 

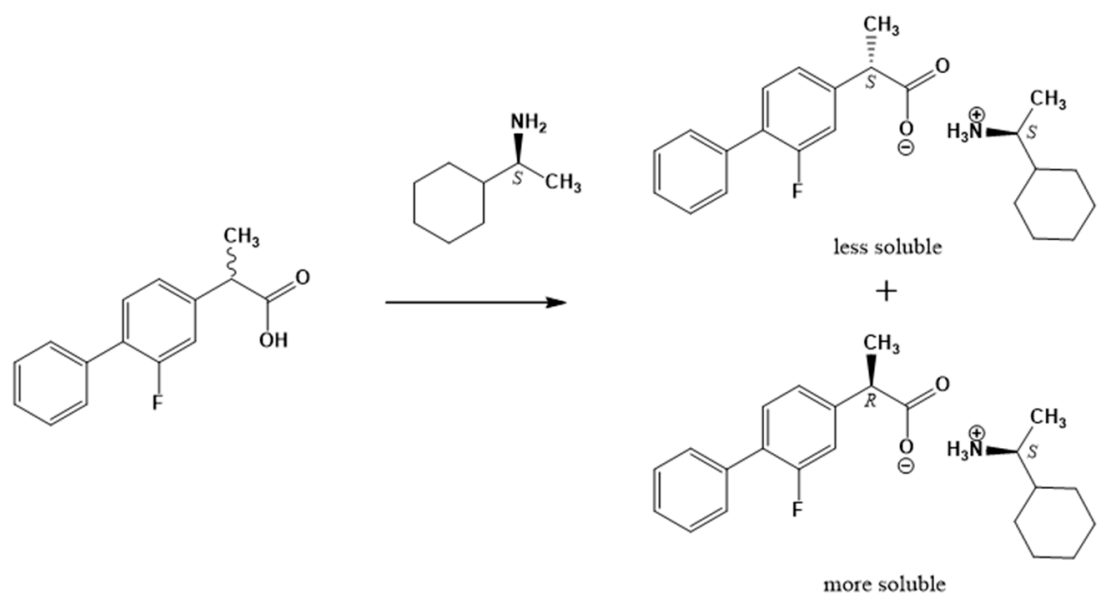

Figure 2. Diastereomeric salt crystallization of flurbiprofen.

\subsection{Solid-State Properties of Flurbiprofen}

An X-ray crystallographic analysis of racemic crystals has been reported [59]; thus, flurbiprofen is a racemic compound under the conditions used to obtain those crystals, although the solvent was not given. Literature generally reports $110-111^{\circ} \mathrm{C}$ (e.g., $\left.[60,61]\right)$ for the melting point (mp) of ( $\mathrm{rac}$ )-flurbiprofen with a high of $113-114{ }^{\circ} \mathrm{C}$ [62-64], while Sigma-Aldrich report $110-112{ }^{\circ} \mathrm{C}$. There is one report [65] of $115-117{ }^{\circ} \mathrm{C}$ (solvent not given) for (S)-flurbiprofen, but this value is highly suspicious of ( $\mathrm{rac}$ )-flurbiprofen given the following results. From Kielce, the mp of ( $\mathrm{rac}$ )-flurbiprofen was recorded as $116-117^{\circ} \mathrm{C}$ $\left(\mathrm{CH}_{2} \mathrm{Cl}_{2}\right)$, while the mp of (S)-flurbiprofen of $97 \%$ ee was recorded as $108-110{ }^{\circ} \mathrm{C}\left(\mathrm{CH}_{2} \mathrm{Cl}_{2}\right)$; from Heidelberg, the $\mathrm{mp}$ of (S)-flurbiprofen ( $97 \%$ ee) was recorded as $109.5-110.5{ }^{\circ} \mathrm{C}$ $\left(\mathrm{CHCl}_{3}\right)$, the $\mathrm{mp}$ of $(\mathrm{R})$-flurbiprofen (commercial material) was recorded as $112-113.5^{\circ} \mathrm{C}$ $\left(\mathrm{CHCl}_{3}\right)$, and the mp of $(\mathrm{rac})$-flurbiprofen was recorded as $117-118.5^{\circ} \mathrm{C}\left(\mathrm{CHCl}_{3}\right)$ with changes-shrinkage and change of refraction-apparent a few degrees before the given $\mathrm{mp}$ range in all three samples that were measured in Heidelberg. Thus, the $\mathrm{mp}$ of the racemate is $\sim 5^{\circ} \mathrm{C}$ higher than that of the enantiomer. In addition to confirming that flurbiprofen is a racemic compound, the very similar mp's of enantiopure and racemic samples suggest that the stabilities of the two crystal structures are close. Because it is expected that volatility correlates with crystal stability, it further suggests that $\mathrm{SDEvS}$ might be difficult to observe with only small differences between the ee's ( $\Delta$ ee's) of the sublimates and residues likely. The magnitude of the SDE is taken as the $\Delta$ ee for various fractions, usually the first and the last.

The infrared (IR) spectra (Figures S1-S7) of (R)-flurbiprofen (commercial material) and (S)-flurbiprofen ( $97 \%$ ee) were essentially identical to each other, with only slight divergences in band intensities being the only apparent differences, and the two spectra were quite distinct from the spectrum of ( $\mathrm{rac}$ )-flurbiprofen, thus further confirming that flurbiprofen is a racemic compound when crystallized from $\mathrm{CHCl}_{3}$. Of particular note, the $v_{\mathrm{OH}}$ for $(\mathrm{rac}$ )-flurbiprofen was markedly altered by a reduction in intensity, a red shift, and splitting into several bands. These changes are consistent with very strong intermolecular hydrogen bonding.

Thus, we confirm that flurbiprofen is a racemic compound by both IR and $\mathrm{mp}$ measurements and, hence, heterochiral association is preferred in the solid state.

A number of intermolecular interactions are conceivable for flurbiprofen, e.g., hydrogen bonding involving acid-acid groups, hydrogen bonding involving acid-fluorine groups, and $\pi-\pi$ stacking. However, by molecular modeling calculations, hydrogen bonding involving only the $-\mathrm{CO}_{2} \mathrm{H}$ groups predominated overwhelmingly over other interactions that involve fluorine bonding for dimeric structures (Tables S2 and S3). Thus, flurbiprofen was considered to be a good test case for the proposed aim of this work, as the hydrogen bonding of the acids groups dominates both intermolecular analyte- 
analyte association (and, thus, also interaction with polar stationary phases), suggesting that elution order might be predictable. Of note, paired structures also only involving hydrogen bonding between $-\mathrm{CO}_{2} \mathrm{H}$ groups were also present in the $\mathrm{X}$-ray crystallographic structure [59].

\subsection{SDEvS}

The very similar mp's that were measured for samples of $(R)_{-},(S)-$, and ( $\mathrm{rac}$ )-flurbiprofen imply that the stabilities of the two crystal structures are also similar and, since it is expected that volatility goes hand-in-hand with stability, sizeable $\Delta e^{\prime}$ 's are consequently not anticipated for SDEvS. Indeed, this was borne out in practice and the SDEvS was difficult to effect. Interestingly, flurbiprofen behaves as a racemic compound by sublimation under quasithermodynamic conditions. For example, sublimation $\left(40{ }^{\circ} \mathrm{C}, 0-0.2 \mathrm{mbar}\right)$ of (S)-flurbiprofen of $59.8 \%$ ee provided a sublimate of $70.4 \%$ ee and a residue of $58.3 \%$ ee after more than $72 \mathrm{~h}$ yielding a $\Delta$ ee of $12.1 \%$, while the sublimation of $(S)$-flurbiprofen of $93.2 \%$ ee under the same conditions provided a sublimate of $85.8 \%$ ee and a residue of $97.8 \%$ ee after $76 \mathrm{~h}$ yielding a $\Delta$ ee of $12.0 \%$. From the various sublimations conducted (Table S4), the euamotic point [66-68] lies between 60-75\% ee. Racemic compound behavior by sublimation has been previously reported for amino acids [66,69] and ibuprofen [70], although, in the latter case, the euamotic point was very high, being close to $90 \%$ ee.

Although the $\triangle \mathrm{ee}^{\prime}$ s for flurbiprofen by SDEvS are not large, there are potential dangers posed by the SDEvS during the preparation, handling, and, particularly, the long-term storage of the drug as SDEvS has been observed during the storage of scalemates with sublimed material in the upper reaches of the container [41,71] as well as with localized partitioning within the sample bulk itself [72]. Even rotary evaporation has been reported [73,74] to effect SDEvS. To test the effect of long-term storage on flurbiprofen, two scalemic samples, ee $87.2 \%$ and $8-9 \mathrm{mg}$ each, were stored in $6 \mathrm{~mL}$ vials at reduced temperatures for six months. One sample that was stored at $+6{ }^{\circ} \mathrm{C}$ incurred a weight loss of $2.8 \%$ with the ee decreasing to $86.5 \%$, while a second sample stored at $-20{ }^{\circ} \mathrm{C}$ incurred a weight loss of $1.3 \%$, with the ee essentially remaining unchanged at $87.4 \%$. Although the alteration in the first sample might be considered inconsequential, the indication for potential problems upon long-term storage is evident.

\subsection{NMR}

NMR examinations of flurbiprofen were conducted for two purposes, to determine the solution-state association preference and use the observations to assess the suitability of the examined solvents for successful chromatographic outcomes with the possibility to also predict the elution order. The assessment of a solvent's suitability for chromatography was based on the intermolecular association observed by NMR and the strength of the SIDA effects. Thus, samples of ( $s c l$ )-flurbiprofen $82 \%$ ee, $(R)$-flurbiprofen, and $(\mathrm{rac})$-flurbiprofen were examined in various solvents $\left(\mathrm{CDCl}_{3}\right.$; toluene- $d_{8}$; acetonitrile- $d_{3} ; 1,4$-dioxane- $d_{8}$; and, cyclohexane- $d_{12}$ (c-hexane- $\left.d_{12}\right)$-methyl tert-butyl ether (MTBE), 4:1) as concentrated $(\sim 5 \mathrm{mg} / 600 \mu \mathrm{L})$ or very concentrated $(\sim 10 \mathrm{mg} / 600 \mu \mathrm{L})$ solutions. The methods that were applied in this work to determine the solution-state association preference by NMR included measurement of $D, T_{1}$, and $T_{2}$. Unfortunately-except for one case-the differences in the $T_{1} \mathrm{~s}$ between the samples of $(R)$-flurbiprofen and ( $\mathrm{rac}$ )-flurbiprofen examined here were very small, as is generally the case, to render a definitive assessment of the preferred solution-state association for the samples for which $T_{1} \mathrm{~s}$ were measured as the measurement error resulted in inconsistencies among the set of signals. Similar problems occurred with the one pair of $T_{2}$ measurements made, with the results being further compounded by $J$ modulation. Table 1 summarizes the NMR results, together with the chromatographic outcomes, which are discussed further on. Detailed descriptions for each of the solvents used are presented below and a catalogue of the NMR examinations is presented in Table S5. 
Table 1. Summary of solution-state association preferences and selected chromatographic outcomes.

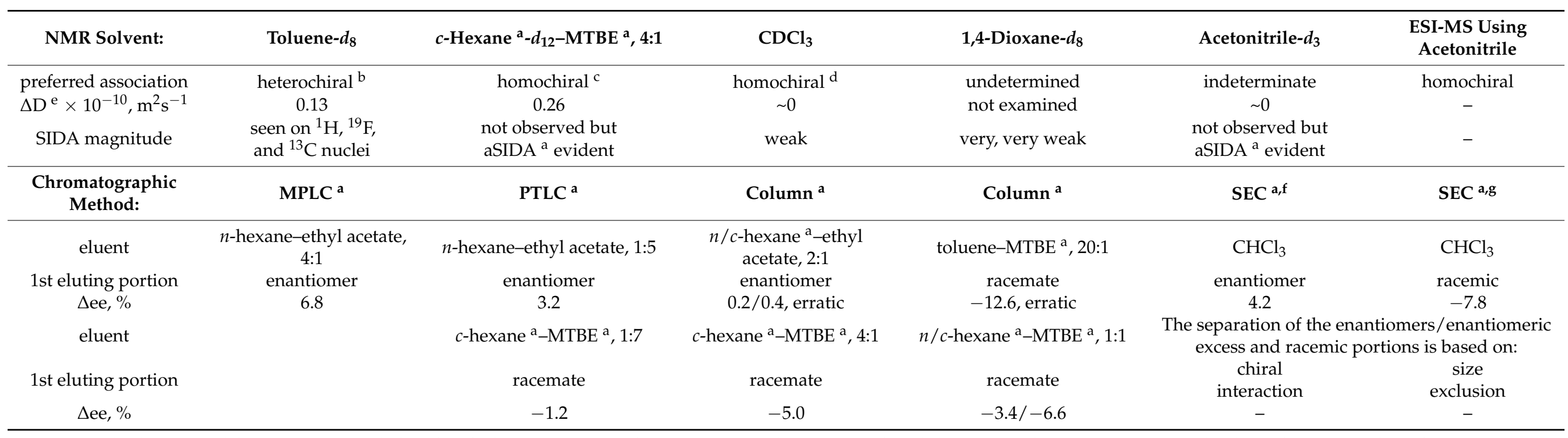

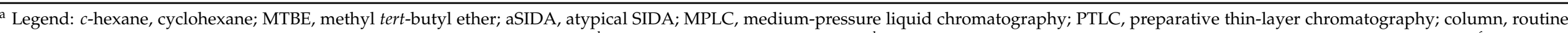

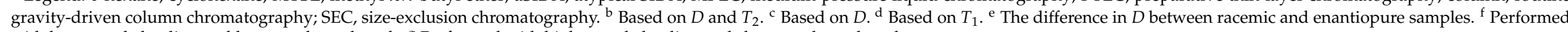
with low sample loading and longer column length. ${ }^{g}$ Performed with high sample loading and shorter column length. 
An examination of ( $R$ )-flurbiprofen and $(\mathrm{rac})$-flurbiprofen samples in acetonitrile- $d_{3}$ failed to reveal significant differences between the two samples for both very concentrated $(21.2 \mathrm{mg} / 660 \mu \mathrm{L})$ and dilute $(2.1 \mathrm{mg} / 600 \mu \mathrm{L})$ samples observing ${ }^{1} \mathrm{H},{ }^{19} \mathrm{~F}$, and ${ }^{13} \mathrm{C}$ nuclei. Clear differences between the concentrated and dilute samples were apparent, indicating intermolecular association, but the differences were small, indicating only a weak association at the higher concentrations. The lack of differentiation, i.e., SIDA, between the two concentrated samples, despite the clear indication of association, could be due to the phenomenon of atypical SIDA (aSIDA), whereby the splitting of signals is not always observed, even though they migrate, although this is dependent on the conditions $[2,5,6]$. In the concentrated samples, the $-\mathrm{CO}_{2} \mathrm{H}$ proton resonated at ca. $9.0 \mathrm{ppm}$ as a very broad signal, while the $-\mathrm{CO}_{2} \mathrm{H}$ carbon resonated at $175.5 \mathrm{ppm}$, with values also being indicative of fairly weak hydrogen bonding. In line with these indications, measurement of $D$ was indeterminate for evaluating the preferred association as the intermolecular association was too low resulting in an insignificant and unreliable difference in $D$, a result that is also in concert with the observed weak aSIDA effects. The much higher values of $D$ for the two samples measured compared to $c$-hexane- $d_{12}-\mathrm{MTBE}(4: 1)$ and toluene- $d_{8}$ also indicated a low degree of intermolecular association.

For a very concentrated sample $(11.3 \mathrm{mg} / 600 \mu \mathrm{L})$ of $(s c l)$-flurbiprofen $82 \%$ ee in $1,4-$ dioxane- $d_{8}$, the conditions again were not conducive to high levels of association and SIDA was not observed for any of the ${ }^{1} \mathrm{H},{ }^{19} \mathrm{~F}$, and ${ }^{13} \mathrm{C}$ nuclei, indicating that association must also be weak, and this was supported by the $-\mathrm{CO}_{2} \mathrm{H}$ proton that resonated at ca. $10.4 \mathrm{ppm}$, although the signal was relatively sharp, and the $-\mathrm{CO}_{2} \mathrm{H}$ carbon that resonated at $175.2 \mathrm{ppm}$. Dilution by a factor of 2 only showed very small or negligible differences in the $\delta^{\prime} \mathrm{s}\left(\Delta \delta^{\prime} \mathrm{s}\right)$ for all ${ }^{1} \mathrm{H},{ }^{19} \mathrm{~F}$, and ${ }^{13} \mathrm{C}$ nuclei, including both the $-\mathrm{CO}_{2} \mathrm{H}$ proton and carbon nuclei. In this case, intermolecular association based on hydrogen bonding may also be with the solvent, rather than just analyte-analyte.

For a concentrated sample $(5.1 \mathrm{mg} / 600 \mu \mathrm{L})$ of $(s c l)$-flurbiprofen $82 \%$ ee in $\mathrm{CDCl}_{3}$, although the conditions were more conducive to higher levels of association, as evidenced by the $-\mathrm{CO}_{2} \mathrm{H}$ proton, which resonated as a broad signal at ca. $11.0 \mathrm{ppm}$, and the $-\mathrm{CO}_{2} \mathrm{H}$ carbon, which resonated at $179.2 \mathrm{ppm}$; surprisingly, SIDA was only observed for the $-\mathrm{CH}_{3}$ protons and not for any of the other ${ }^{1} \mathrm{H},{ }^{19} \mathrm{~F}$, and ${ }^{13} \mathrm{C}$ nuclei. Very concentrated samples $(10.1 \mathrm{mg} / 600 \mu \mathrm{L})$ of $(R)$-flurbiprofen and ( $\mathrm{rac}$ )-flurbiprofen revealed only very small $\Delta \delta^{\prime} \mathrm{s}$ to each other and to the scalemic sample for the ${ }^{1} \mathrm{H}$ nuclei; for the ${ }^{19} \mathrm{~F}$ nuclei, only a negligible $\Delta \delta$ between the very concentrated $(R)$-flurbiprofen and ( $\mathrm{rac}$ )-flurbiprofen samples was observed, but a small difference for these two samples to the concentrated scalemic sample was observed; for the ${ }^{13} \mathrm{C}$ nuclei, the $\Delta \delta^{\prime}$ 's between the very concentrated $(R)$-flurbiprofen and ( $r a c)$-flurbiprofen samples were either very small or non-existent, and similarly for the comparison to the concentrated scalemic sample. An increased association in the very concentrated $(R)$-flurbiprofen and ( $\mathrm{rac}$ )-flurbiprofen samples was evident by the $-\mathrm{CO}_{2} \mathrm{H}$ proton, which resonated as a broad signal at ca. $11.3 / 2 \mathrm{ppm}$, and the $-\mathrm{CO}_{2} \mathrm{H}$ carbon, which resonated at $179.8 \mathrm{ppm}$. The measurement of $D$ was indeterminate for evaluating the preferred association as the intermolecular association was again too low, resulting in an insignificant and unreliable difference in $D$, a result that is also in concert with the observed weak SIDA effects. The much higher values of $D$ for the two samples measured as compared to $c$-hexane- $d_{12}-\operatorname{MTBE}(4: 1)$ and toluene- $d_{8}$ also indicated a low degree of intermolecular association and intermolecular association based on hydrogen bonding may also again be with the solvent. Very surprisingly, though, the measurement of the $T_{1} \mathrm{~s}$ for the very concentrated $(R)$-flurbiprofen and ( $\mathrm{rac}$ )-flurbiprofen samples yielded a consistent indication for homochiral preference across the set of spins. As a consequence, the expectation is that, for SDE via SEC using $\mathrm{CHCl}_{3}$ as eluent, the enantiomeric excess portion would elute first, although the $\Delta$ ee would unlikely be sizeable due to a low degree of association.

For a concentrated sample $(5.1 \mathrm{mg} / 600 \mu \mathrm{L})$ of $(s c l)$-flurbiprofen $82 \%$ ee in $c$-hexane- $d_{12}$ MTBE (4:1), the conditions again were still not conducive to high levels of association and 
SIDA was not observed for any of the ${ }^{1} \mathrm{H},{ }^{19} \mathrm{~F}$, and ${ }^{13} \mathrm{C}$ nuclei indicating that association must also be weak, and this was confirmed by the $-\mathrm{CO}_{2} \mathrm{H}$ proton, which resonated at ca. $9.0 \mathrm{ppm}$ as a broad signal, and the $-\mathrm{CO}_{2} \mathrm{H}$ carbon, which resonated at $174.8 \mathrm{ppm}$. For a very concentrated sample $(10.7 \mathrm{mg} / 600 \mu \mathrm{L})$ of $(s c l)$-flurbiprofen $82 \%$ ee, although SIDA was still not observed for any of the ${ }^{1} \mathrm{H},{ }^{19} \mathrm{~F}$, and ${ }^{13} \mathrm{C}$ nuclei, there was a clear indication of association, as practically all of the ${ }^{1} \mathrm{H},{ }^{19} \mathrm{~F}$, and ${ }^{13} \mathrm{C}$ signals exhibited small, but significant, $\Delta \delta^{\prime}$ s, especially the $-\mathrm{CO}_{2} \mathrm{H}$ proton, which resonated at now ca. $9.9 \mathrm{ppm}$ and as a relatively sharp signal, and the $-\mathrm{CO}_{2} \mathrm{H}$ carbon, which now resonated at $175.3 \mathrm{ppm}$. However, a comparison of more concentrated $(8.1 \mathrm{mg} / 600 \mu \mathrm{L})$ samples of $(R)$-flurbiprofen and ( $\mathrm{rac}$ )-flurbiprofen did not reveal clear $\delta$ differences between the two samples for ${ }^{1} \mathrm{H}$, ${ }^{19} \mathrm{~F}$, and ${ }^{13} \mathrm{C}$ nuclei. Similarly for a pair of very concentrated $(10.4 \mathrm{mg} / 600 \mu \mathrm{L})$ samples of (R)-flurbiprofen and ( $\mathrm{rac}$ )-flurbiprofen. The lack of $\delta$ differentiation, i.e., SIDA, between the two concentrated samples, despite the clear indication of association, could again be due to aSIDA. The measurement of the $T_{1} \mathrm{~s}$ for the very concentrated $(R)$-flurbiprofen and ( $\mathrm{rac}$ )-flurbiprofen samples yielded inconsistencies across the set of spins and, thus, an indication for associate preference was not forthcoming. However, the measurement of $D$ clearly indicated that homochiral association was preferred in the solvent system $c$ hexane- $d_{12}-\mathrm{MTBE}$ (4:1). As a consequence, the expectation is that for SDE via normal-phase chromatography using eluents predominantly consisting of $c$-hexane, the enantiomeric excess portion would elute first.

For a concentrated sample $(5.3 \mathrm{mg} / 600 \mu \mathrm{L})$ of $(s c l)$-flurbiprofen $82 \%$ ee in toluene- $d_{8}$, a reasonable association was evidenced by the $-\mathrm{CO}_{2} \mathrm{H}$ proton, which resonated as a very broad signal at ca. $10.4 \mathrm{ppm}$, and good association by the $-\mathrm{CO}_{2} \mathrm{H}$ carbon, which resonated at $180.0 \mathrm{ppm}$. While SIDA was clearly observed for the $-\mathrm{CH}_{3}$ protons (Figure 3 ) and the fluorine (Figure 4), it could not be observed for the $-\mathrm{CH}$ proton, and, while it could be seen on some aromatic protons signals, it was obscured by the complexity of the multiplets for most of them. Only for a few ${ }^{13} \mathrm{C}$ nuclei could SIDA be observed, e.g., C-4 (Figure 5). As expected, for very concentrated samples of $(10.8 \mathrm{mg} / 600 \mu \mathrm{L})$ of $(R)$-flurbiprofen and (rac)-flurbiprofen, an increased association was evident by the $-\mathrm{CO}_{2} \mathrm{H}$ proton, which resonated as a very broad signal at ca. $10.6 \mathrm{ppm}$, and the $-\mathrm{CO}_{2} \mathrm{H}$ carbon, which resonated at $180.4 \mathrm{ppm}$. As before, for these very concentrated samples, only very small $\Delta \delta^{\prime}$ s to each other were observed and also to the concentrated scalemic sample for the ${ }^{1} \mathrm{H}$ nuclei while clearer differences were apparent for the ${ }^{19} \mathrm{~F}$ nuclei between all three samples. For the ${ }^{13} \mathrm{C}$ nuclei, the $\Delta \delta$ 's between the very concentrated $(R)$-flurbiprofen and ( $\mathrm{rac}$ )-flurbiprofen samples were either very small or non-existent, and generally also for comparison to the concentrated scalemic sample, but with a few exceptions. However, the measurement of the $T_{1}$ s for the very concentrated $(R)$-flurbiprofen and $(\mathrm{rac})$-flurbiprofen samples yielded inconsistencies across the set of spins and, thus, an indication for associate preference was not forthcoming. Despite $J$ modulation voiding almost all of the $T_{2}$ measurements, one spin was sufficiently resilient to $J$ modulation, wherein the values of $T_{2}$ for H-9 indicated that heterochiral was the preferred associate. Finally, the measurement of $D$ clearly indicated that heterochiral association was indeed preferred in toluene- $d_{8}$. As a consequence, the expectation is that for SDE via normal-phase chromatography using eluents predominantly consisting of toluene, the racemic portion would elute first.

While the signal of the $-\mathrm{CO}_{2} \mathrm{H}$ proton does give a reasonable indication of intermolecular association, whether it be analyte-analyte or analyte-solvent, often it is too broad for identification of the solution-state association preference, homo- or heterochiral, if analyte-analyte association is involved and, additionally, is sensitive to variations in such parameters as the $\mathrm{pH}$ and the water content. The signal of the $-\mathrm{CO}_{2} \mathrm{H}$ carbon is more reliable in this regard, although it suffers from the obvious limitation of reduced sensitivity. Unfortunately, insufficient differences were found to affect the identification of the homoor heterochiral solution-state association preference for the samples examined here based on the $\delta^{\prime}$ s of these nuclei. 


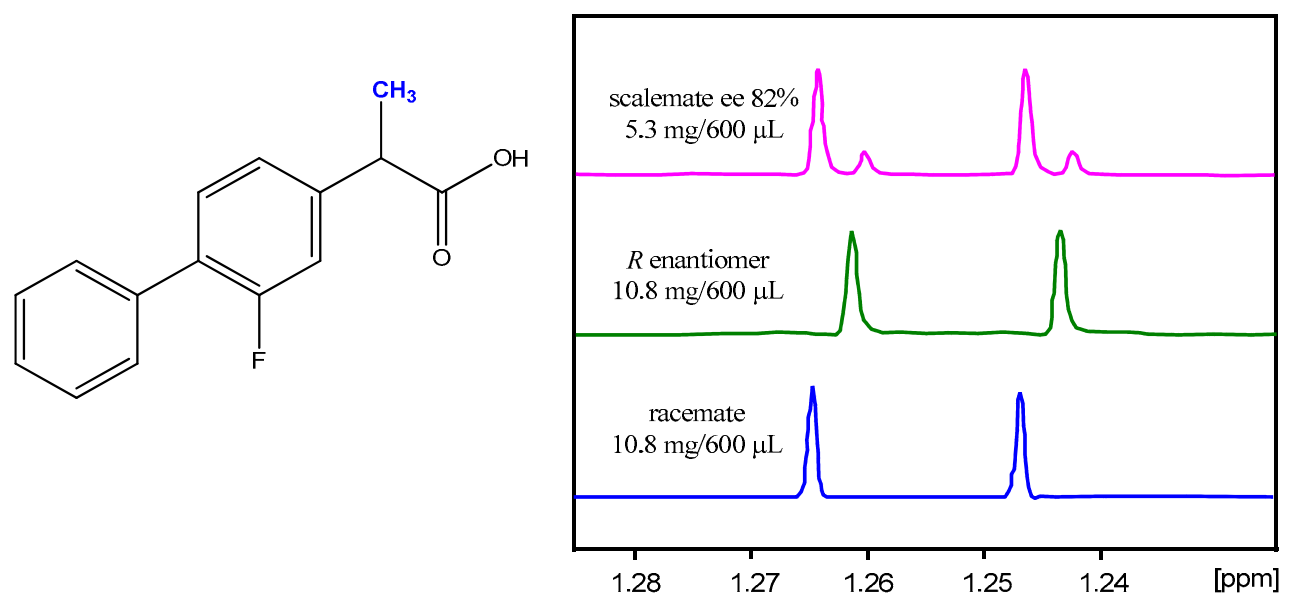

Figure 3. The ${ }^{1} \mathrm{H}$ nuclear magnetic resonance (NMR) signal of the $-\mathrm{CH}_{3}$ protons in toluene- $d_{8}$ at $25{ }^{\circ} \mathrm{C}$ for very concentrated $(R)$-flurbiprofen and $(\mathrm{rac})$-flurbiprofen samples and for a concentrated sample of $(s c l)$-flurbiprofen $82 \%$ ee. Spectra were processed applying a double exponential to the FID prior to Fourier transformation.<smiles>CC(C(=O)O)c1ccc(-c2ccccc2)c(F)c1</smiles>

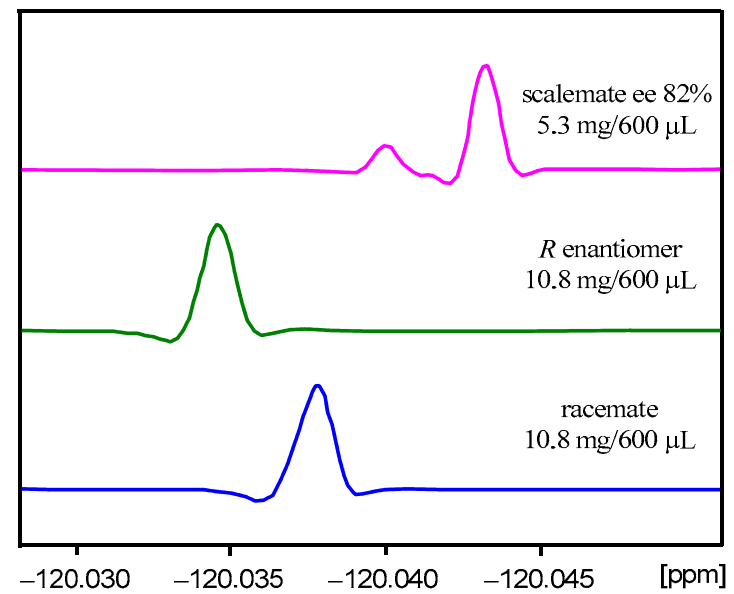

Figure 4. The ${ }^{19} \mathrm{~F}$ NMR signal of the fluorine nucleus in toluene- $d_{8}$ at $25^{\circ} \mathrm{C}$ for very concentrated $(R)$ flurbiprofen and ( $\mathrm{rac}$ )-flurbiprofen samples and for a concentrated sample of $(\mathrm{scl})$-flurbiprofen $82 \%$ ee. Spectra were processed applying a double exponential to the FID prior to Fourier transformation.

Spins that only exhibit $\Delta \delta^{\prime}$ s due to intermolecular association by electronic changes manifested through the bonds and are, thus, ostensibly indifferent to the nature of the association are more likely to be reliable probes for evaluation of the association preference. Thus, ironically, spins further from the site of intermolecular interaction are likely to fulfill this criterion, since they will not experience stereo-electronic or steric interactions due to intermolecular association with $\delta$ trends that may be in opposition. Additionally, for spins close to the site of association-either through bonds or through space-the $\Delta \delta^{\prime}$ 's due to intermolecular association may even be much larger for the less favored associate due to the differential effects of the stereo-electronic or steric interactions between the diastereomeric associates. Surprisingly, for flurbiprofen, the effects of intermolecular association could be seen well away from the site of association, e.g., even on the meta protons of the second phenyl group, the H-12s. Indeed, $\Delta \delta^{\prime}$ s were very often even more apparent on the relatively distant ortho and meta protons of the first phenyl group, $\mathrm{H}-5, \mathrm{H}-8$, and $\mathrm{H}-9$, than they were on the $-\mathrm{CH}_{3}$ protons, $\mathrm{H}-3$, and the $-\mathrm{CH}$ proton, $\mathrm{H}-2$. Unfortunately, in these examinations, the $\Delta \delta^{\prime}$ s for the distal nuclei were also considered to be too small to enable differentiation of the associate preference. 


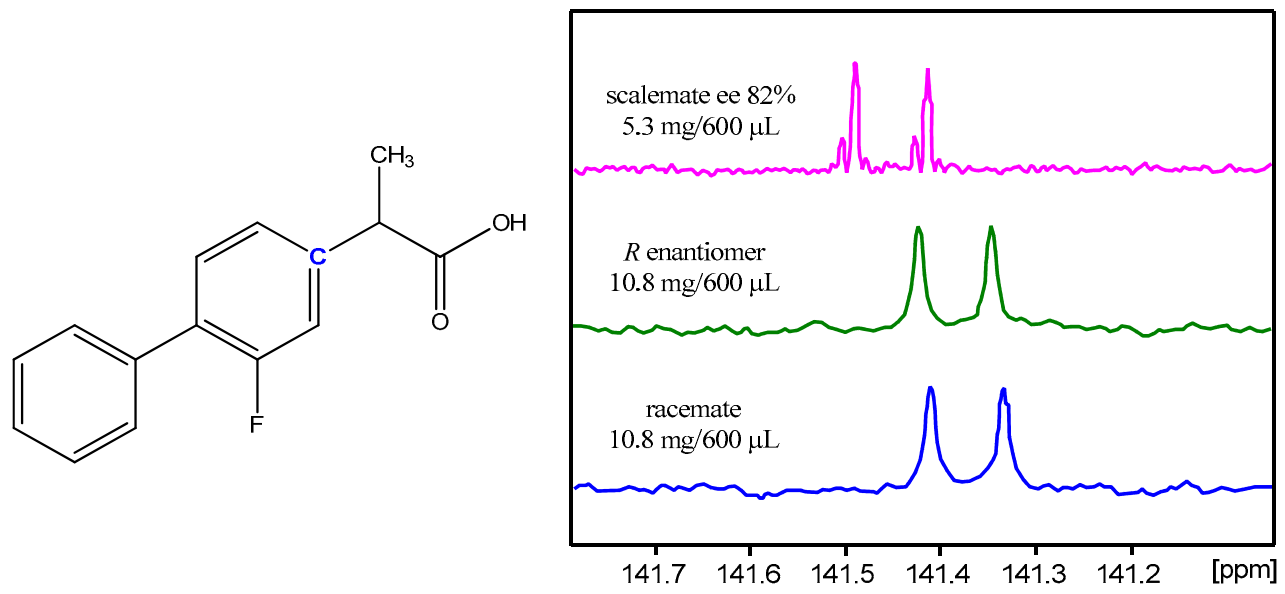

Figure 5. The ${ }^{13} \mathrm{C}$ NMR signal of $\mathrm{C}-4$ in toluene- $d_{8}$ at $25{ }^{\circ} \mathrm{C}$ for very concentrated $(R)$-flurbiprofen and (rac)-flurbiprofen samples and for a concentrated sample of ( $s c l$ )-flurbiprofen $82 \%$ ee. The spectrum of the $(s c l)$-flurbiprofen sample was processed applying a double exponential to the FID prior to Fourier transformation while the spectra of the $(R)$-flurbiprofen and ( $r a c)$-flurbiprofen samples were processed applying a single exponential decay to the FID prior to Fourier transformation.

\subsection{SDEvC}

Flurbiprofen was considered to be a good test case for predicting the elution order as the principal analyte-stationary phase interaction for chromatography over silica is assumed to be the hydrogen bonding of the acid group, but, if this is tied up with dimeric association, then the preferred associate should elute first. Thus, it was hoped that the elution order might simply follow the solution-state association preference as determined by NMR. However, negligible and inconsistent results were obtained for routine gravitydriven column (column) chromatography over silica gel using $n / c$-hexane-ethyl acetate or $n$-hexane-MTBE as eluent (Table 1, Tables S6-S8, and entries 1-3 in Table S18), and, although the elution order was in accordance with expectations for the $n / c$-hexane-ethyl acetate (enantioenriched initial fractions), a reverse elution order was observed for $n$ hexane-MTBE, contrary to expectations. A change in elution order upon a change of solvent has been previously reported [75], although it is a relatively uncommon occurrence. Only when using c-hexane-MTBE (either in a 1:1 or 4:1 ratio) were small and consistent SDEs observed with $\Delta$ ee's of -6.6 and $5.0 \%$, respectively (Table 1, Tables S9 and S10, and entries 4 and 5 in Table S18). The small $\Delta$ ee's are unsurprising, given the results of the NMR analysis using $c$-hexane- $d_{12}$-MTBE (4:1), whereby SIDA was very weak for (scl)flurbiprofen. Stronger effects were obtained when using toluene-MTBE (20:1) with a $\Delta$ ee of $-12.6 \%$, and with an elution order at least consistent with the NMR-determined preference for heterochiral association in toluene- $d_{8}$ solution (Table 1, Table S11, and entry 6 in Table S18). A cause for the elution order anomalies could a change in associate preference within the very different environment of the stationary phase pores relative to the interstitial voids. In addition, the mixed solvent systems in use might lead to a change in the solvent composition within the pores of the stationary phase, thus also affecting the associate preference. Because the $\Delta$ ee's are quite small, it is entirely plausible that this is the case.

Because the SDE magnitude for SDEvC has been postulated to increase as the system is scaled down [76], (scl)-flurbiprofen was subjected to both medium-pressure liquid chromatography (MPLC) and preparative thin-layer chromatography (PTLC) (Table 1, Tables S12-S14, and entries 7-11 in Table S18). Although erratic results were again obtained to a degree, larger $\Delta$ ee's were indeed observed for $n$-hexane-ethyl acetate eluent mixtures, e.g., for MPLC using $n$-hexane-ethyl acetate (2:1) as eluent, a $\Delta$ ee of $6.8 \%$ was obtained while for PTLC using $n$-hexane-ethyl acetate (1:5) as eluent, a $\Delta$ ee of $3.2 \%$ was obtained. Using $c$-hexane-MTBE (1:7) as eluent for PTLC, a $\triangle$ ee of $-1.2 \%$ was obtained, i.e., the reverse order of elution occurred, as per the column chromatographic run. However, in 
this case, scaling down did not increase the SDE, but the performance of the PTLC may have been compromised by the high concentration of MTBE that is required to affect the migration of the flurbiprofen.

The preference, albeit minor, for homochiral association in $\mathrm{CDCl}_{3}$, as determined by $\mathrm{NMR}$, was expected to lead to an elution order for SEC using $\mathrm{CHCl}_{3}$ as the eluent, whereby the initial fraction(s) would be enantioenriched and the later fraction(s) enantiodepleted, since the preferred associate would average a higher molecular weight and, thus, would elute faster. This initially seemed to be the case as the first run (Table 1, Table S15, and entry 12 in Table S18) with a low loading and the long column did indeed provide enantioenriched initial fractions as expected, although with only a minor $\Delta$ ee of 4.2 ee and a somewhat erratic elution profile. However, subsequent runs (Table 1, Tables S16 and S17, and entries 13 and 14 in Table S18) with higher loadings and shorter columns (thus, with less progressive dilution along the column) provided the opposite elution order. This switch in elution order due to the latter conditions is inconsistent with SDE via SEC as the predominate process in the initial run and another process, viz. chiral chromatography, must be in effect. With SEC as the predominate process for the entries 13 and 14 in Table S18 with entry 14 of shorter column length but with the same loading as entry 13 , it is in line with expectations that the $\Delta$ ee is larger for entry 14 (-7.8 vs. -4.4 ee), as chiral chromatography only competes with SEC as the column lengthens due to the diminishing effect of SEC as a result of progressive dilution along the column. The anomalous elution order for entries 13 and 14 could be due to a change in associate preference within the very different environment of the stationary phase pores relative to the interstitial voids. Of note, SDE via SEC has been previously reported [34] using polystyrene gel as the stationary phase and $\mathrm{CDCl}_{3}$ as the eluent and where a high bias for the homochiral associate provided an elution order consistent with expectations.

\subsection{ESI-MS}

Because, by NMR, is was difficult and even not possible to definitively assess the solution-state association preference in polar solvents due to diminished association, mass spectra using ESI as the method of ionization was utilized to provide an indication of the preferred solution-state association. ESI spectra of solutions of (S)-flurbiprofen (97\% ee), ( $s c l$ )-flurbiprofen (75\% ee), and ( $\mathrm{rac}$ )-flurbiprofen in acetonitrile, each at concentrations of $0.3,0.4$, and $0.5 \mathrm{mg} / \mathrm{mL}$, were acquired using ionization energies that ranged from $0.5-9 \mathrm{eV}$ in negative-ion mode from which the ion intensities of the predominate $[2 \mathrm{M}-\mathrm{H}]^{-}$dimer ion (Figures S8 and S9) were plotted against the ionization energy for each concentration (Figure 6, Figures S10-S12). At each concentration, clear differences between the plots for the three samples were readily observable (aside from the $0.3 \mathrm{mg} / \mathrm{mL}$ solutions, where the difference in plots between ( $s c l)$-flurbiprofen $(75 \%$ ee) and ( $\mathrm{rac}$ )-flurbiprofen was not perceptible, presumably due to the low ion counts). Because the ion counts directly reflect the degree of association in the droplets within the ESI source as the solvent is stripped away (neglecting any contribution from the stability of the dimeric ions once formed in the gaseous phase), homochiral dimeric associates are clearly favored in acetonitrile solution, in line with the higher polarity of the solvent relative to toluene and $n / c$-hexane (Table 1 ). 


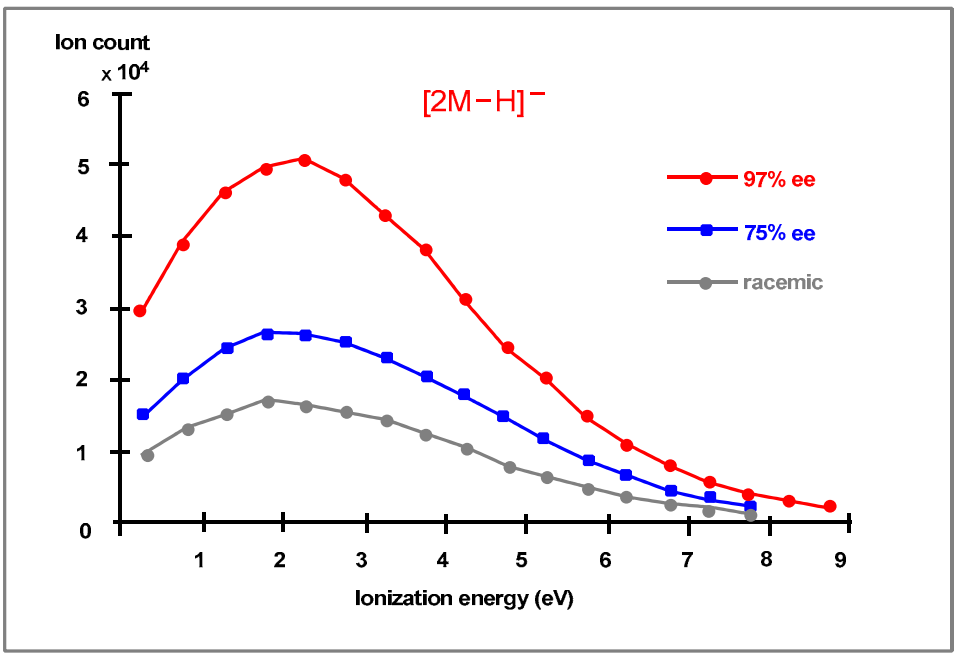

Figure 6. Ion counts for the $[2 \mathrm{M}-\mathrm{H}]^{-}$dimer ion vs. ionization energy for the $0.5 \mathrm{mg} / \mathrm{mL}$ solutions.

\section{Conclusions}

Flurbiprofen, a racemic compound, was found to exhibit SDE by chromatographycolumn chromatography, MPLC, PTLC, and SEC-and by sublimation. Examination by NMR in various solvents found that flurbiprofen also exhibited the phenomenon of SIDA. By the measurement of $D, T_{1}$, and $T_{2}$ using NMR, as well as by ESI-MS examinations, the preferred intermolecular association was found to be solvent-dependent, e.g., heterochiral association was preferred in toluene, while homochiral association was preferred in more polar solvents. While it was possible to determine the solution-state association preference by NMR relatively easily, the desired correlation between NMR observations and chromatographic outcomes was not forthcoming, thus precluding the possibility of predicting chromatographic outcome based on NMR observations. The expectation was that flurbiprofen would be an ideal test molecule for this idea, since the intermolecular interactions of flurbiprofen are overwhelmingly dominated by hydrogen bonding involving only the carboxylic groups and, although this expectation was not met, the behavior of scalemic flurbiprofen was much more interesting and perhaps even more revealing. Failure to predict the chromatographic outcomes was ostensibly due to the switch in association preference, depending on the conditions, e.g., environment polarity. The switch from hetero- to homochiral association preference not only negates the possibility to predict the elution order, but also precluded a strong $\triangle \mathrm{ee}$ for SDEvC. However, SDEvC behavior is clearly quite complex and subject to subtle influences and it cannot be reduced to simple parameterization, as postulated in this instance, but it may be possible in cases where the $\mathrm{SDE}$ is much greater. In concert with the close mp's of enantiopure and racemic crystals, SDEvS was found to be difficult to effect, but nevertheless observable, even for the extended storage of samples at low temperatures.

SDEvC and SDEvS both have ramifications for the preparation, handling, and storage of scalemic samples of flurbiprofen, and this concern also applies to other chiral drugs since the SDE always clearly occurs to some degree, even in unfavorable systems and the potential for unintended detrimental effects due to the inherent dangers of the SDE must never be ignored or underestimated. Of wider and greater significance, this first comprehensive study of the SDE properties of a marketed fluorinated drug opens up a new line of enquiry that should be pursued by industrial and academic research laboratories [77]. Thus, when considering the ever growing number of fluorine-containing drugs on the pharmaceutical market [78-83], the importance of including the SDE for a complete description of the physicochemical and biological [84] properties of a modern pharmaceutical can hardly be ignored. 


\section{Experimental}

4.1. General

(rac)-Flurbiprofen was purchased from Nine-Dinn Chemistry, Shanghai, China. In addition to the enantioenriched samples that were prepared from ( $\mathrm{rac}$ )-flurbiprofen, a commercial sample of $(R)$-flurbiprofen was also purchased from Cayman Chemical, Ann Arbor, USA.

\subsection{Preparation of Enantioenriched Flurbiprofen}

Enzymatic enantioresolution of ( $(r a c)$-flurbiprofen to (S)-flurbiprofen was performed according to [56], but, briefly: to (rac)-flurbiprofen $(1 \mathrm{~g})$ in $c$-hexane $(25 \mathrm{~mL})$, methanol $(0.66 \mathrm{~mL})$, and DMF $(0.11 \mathrm{~mL})$ was added acrylic resin containing Candida antarctica lipase $(0.27 \mathrm{~g})$. The reaction mixture was stirred at $45^{\circ} \mathrm{C}$ while monitoring the progress of the reaction and the ee of unreacted flurbiprofen by chiral HPLC that was unable to resolve the flurbiprofen methyl ester enantiomers. Aliquots for HPLC analysis were taken after $1(44.4 \%$ ee, conversion $32 \%), 2$ ( $86 \%$ ee, conversion $53 \%$ ), and $3(77 \%$ ee, conversion $66 \%$ ) days. The flurbiprofen methyl ester ee was determined after acidic hydrolysis and only once after two days of reaction, at which time the ee of the (R)-flurbiprofen was $58 \%$. After three days the acrylic resin was filtered off and the solvents removed under vacuum. The mixture of $(S)$-flurbiprofen and $(R)$-flurbiprofen methyl ester was separated and concomitantly purified by column chromatography over silica gel using $n$-hexaneethyl acetate (3:1) as eluent initially and, then, following elution of the ester, in a 1:1 ratio to provide $0.36 \mathrm{~g}$ of $(S)$-flurbiprofen and $0.59 \mathrm{~g}$ of $(R)$-flurbiprofen methyl ester.

Diastereomeric salt enantioenrichment was performed with modification according to [57], but briefly: to flurbiprofen $(200 \mathrm{mg})$ in ethanol $(5 \mathrm{~mL})$ was added a chiral amine ( 0.5 equiv. of either 1-cyclohexyl ethylamine or 1-phenyl ethylamine), which resulted in the formation of a white precipitant. The suspension was heated until all of the solid had dissolved and the solution left overnight at room temperature. The white solid that had precipitated was filtered off and washed with cold ethanol. The diastereomeric salt was decomposed using $1 \mathrm{M} \mathrm{HCl}$ and the free acid was extracted with $\mathrm{CH}_{2} \mathrm{Cl}_{2}$. The removal of the solvent under vacuum yielded enantioenriched flurbiprofen. Table S1 tabulates various runs.

\section{3. $N M R$}

The NMR spectra were acquired at $25^{\circ} \mathrm{C}$ using Bruker $14.1 \mathrm{~T}$ Avance and 9.4 T Avance III NMR spectrometers operating at 600 and $400 \mathrm{MHz}$, respectively, for ${ }^{1} \mathrm{H}$ nuclei, 150 and $100 \mathrm{MHz}$, respectively, for ${ }^{13} \mathrm{C}$ nuclei, and $376 \mathrm{MHz}(9.4 \mathrm{~T})$ for ${ }^{19} \mathrm{~F}$ nuclei. Samples of flurbiprofen were acquired in $\mathrm{CDCl}_{3}$, toluene- $d_{8}, 1,4$-dioxane- $d_{8}$, acetonitrile- $d_{3}$, and $c$-hexane- $d_{12}-$ MTBE (4:1) at the indicated concentrations (Table S5) and, if needed, the concentration of one sample of a pair was adjusted with additional solvent, such that the ${ }^{1} \mathrm{H}$ signal intensities of the two samples matched. Solvent signals (tetramethyl silane in the case of $\mathrm{CDCl}_{3}$ ) were used for chemical shift calibration and to substantiate the veracity of the experimental results by confirming a lack of significant changes between enantiopure and racemic samples for the measured parameter or adjusting accordingly. The measurements of $D$ were made without sample spinning using the bipolar pulse pair longitudinal eddy current delay (BPPLED) sequence [85] employing half-sinusoidal gradient pulses. The gradient strength was incremented linearly in 32 steps from 0.828 to $40.549 \mathrm{G} / \mathrm{cm}$; the diffusion delay big delta, $\Delta$, was set to $19.75 \mathrm{~ms}$; little delta, $\delta$, to $4.4 \mathrm{~ms}$; the gradient pulses to $1.1 \mathrm{~ms}$; the eddy current delay, $T e$, to $5 \mathrm{~ms}$; the $A q$ and post-acquisition delay (PAD) times together totaled $13.3 \mathrm{~s}$; and, the number of scans per gradient increment was 16. The effect of convection was checked by also measuring the samples in $2.5-\mathrm{mm}$ tubes and comparison with results obtained from measurements on samples in 5-mm tubes. Since the effect of convection was found to have no impact on the order and only minimal impact on the relative magnitude of the $D^{\prime} \mathrm{s}, 5-\mathrm{mm}$ tubes were generally used for convenience. The longitudinal relaxation times, $T_{1}$, were measured using demagnetization- 
recovery [86], with 32 recovery times within the range $0.001-50 \mathrm{~s}$. The number of scans per recovery time increment was 8 . Transverse relaxation times, $T_{2}$, were measured using the Carr-Purcell-Meiboom-Gill (CPMG) pulse sequence with 16 decay times within the range $0.02-12 \mathrm{~s}$ using echo times of $10 \mathrm{~ms}$. The number of scans per decay time increment was 8 . The numerical values for $D, T_{1}$, and $T_{2}$ were calculated based on both area and peak intensity using curve-fitting procedures available in the standard Bruker software package TopSpin 2.1 and 3.6. The ${ }^{13} \mathrm{C}$ NMR spectra were processed without any applied line broadening, application of $1 \mathrm{~Hz}$ line broadening (single exponential decay), or with a double exponential as necessary to observe closely resonating signals.

\subsection{IR}

The IR spectra were recorded using a Bruker LUMOS instrument that was equipped with a germanium crystal for ATR measurements. To ensure that the samples were directly comparable, several mgs of each of the prepared (S)-flurbiprofen ( $97.5 \%$ ee), commercial $(R)$ flurbiprofen, and (rac)-flurbiprofen were dissolved in 1-2 $\mathrm{mL}$ of $\mathrm{CHCl}_{3}$, and the solutions were allowed to dry in open vials on the bench for a few days. These samples were also used to measure mp's.

\subsection{Chromatography}

Column chromatography was performed over silica gel using a column of dimensions $1.5 \times 40 \mathrm{~cm}$. The solvent mix for entries 1-4 in Table S18 was selected on the basis of TLC by searching for a solvent mix that provided an $R_{\mathrm{f}}$ ca. 0.2 . Scalemic samples of $100 \mathrm{mg}$ that were enriched in the $S$ enantiomer were loaded onto the column as a solution in the eluent. $10 \mathrm{~mL}$ fractions were generally collected and the ee's of the collected fractions were determined by chiral HPLC. SEC was conducted using Sephadex LH-20 as the stationary phase packed into a column of dimensions $1.5 \times 40 \mathrm{~cm}$ eluting with $\mathrm{CHCl}_{3}$. MPLC was performed on an Isolera One Biotage instrument that was equipped with a silica gel column (grain size $20 \mu \mathrm{m}$ ) using a flow rate of 5 or $2 \mathrm{~mL} / \mathrm{min}$. $10 \mathrm{~mL}$ fractions were collected and the ee's of the collected fractions determined by chiral HPLC. PTLC was performed using silica gel $60 \mathrm{~F}_{254}$ plates of dimensions $20 \times 20 \times 0.2 \mathrm{~cm}$ using various eluents (Table S14). After development, the bands were fractioned, the analyte desorbed from the stationary phase, and the ee's of the fractions determined by chiral HPLC. HPLC analysis was conducted using UV-vis detection at $\lambda=254 \mathrm{~nm}$ and $n$-hexane-isopropanol (19:1) as eluent at a flow rate of $0.8 \mathrm{~mL} / \mathrm{min}$. Chiral HPLC analysis was conducted using a Lux $5 \mathrm{~mm}$ Amylose- 1 column $(5 \mu \mathrm{m}, 250 \times 4.6 \mathrm{~mm})$ and $n$-hexane-isopropanol (90:10) as eluent at a flow rate of $0.8 \mathrm{~mL} / \mathrm{min}$. with UV-vis detection at $\lambda=254 \mathrm{~nm}$. Retention times: $R$ enantiomer, 9.85 min.; $S$ enantiomer, 11.96 min. (Figures S16 and S17).

\subsection{ESI-MS}

The ESI mass spectra (Figures S8 and S9) were acquired using a Bruker micrOTOF-Q II that was equipped with a syringe pump. The drying gas flow rate was $4.0 \mathrm{~L} / \mathrm{min}$. heated to $200{ }^{\circ} \mathrm{C}$ and the capillary voltage was $4500 \mathrm{~V}$, while the ionization energy was varied over the range $0.5-9 \mathrm{eV}$. Samples of (S)-flurbiprofen ( $97 \% \mathrm{ee)}$ ), ( $\mathrm{scl}$ )-flurbiprofen ( $75 \%$ ee), and (rac)-flurbiprofen in acetonitrile, each at concentrations of $0.3,0.4$, and $0.5 \mathrm{mg} / \mathrm{mL}$, were acquired using ionization energies that ranged from $0.5-9 \mathrm{eV}$ in negative-ion mode (Figures S10-S12).

\subsection{Sublimation}

Purified material from enzymatic enantioresolution reactions was used for the sublimation tests. Prior to use, the material was thoroughly mixed and ground to provide powdered samples. Sublimations were performed under either ambient pressure or at pressures within the range of $0-0.2$ mbar at various temperatures in the range $40-100{ }^{\circ} \mathrm{C}$. Table S4 compiles the results. 
Supplementary Materials: The following are available online at https://www.mdpi.com/2073 -8994/13/4/543/s1: IR spectra, diastereomeric salt crystallization results, molecular modeling results, sublimation results, catalogue of NMR experiments, SDEvC tables and plots, summary of chromatographic results, MS and ion counts vs. ionization energy plots, NMR spectra, and chiral HPLC traces.

Author Contributions: Conceptualization, A.W., V.A.S., K.D.K.; methodology, A.W., M.K., K.D.K., V.A.S.; formal analysis, A.W., M.K., K.D.K., V.A.S.; investigation, A.W., M.K., M.U., A.K., K.D.K.; resources, A.W., J.H., M.K., K.D.K.; data curation, M.K., A.W., K.D.K., V.A.S.; writing-original draft preparation, A.W., K.D.K.; writing-review and editing, V.A.S., K.D.K., J.H.; supervision, K.D.K., V.A.S.; project administration, V.A.S., K.D.K. All authors have read and agreed to the published version of the manuscript.

Funding: The authors gratefully acknowledge financial support from the Ministry of Science and Higher Education, Poland (grant no. 668, A.W.; grant no. 659, M.K.; and SMGR.RN.20.264, A.K.) and IKERBASQUE, the Basque Foundation for Science, Spain (V.A.S.).

Acknowledgments: Petra Krämer from the Organic Chemistry Institute, University of Heidelberg is thanked for acquiring the IR spectra.

Conflicts of Interest: The authors declare no conflict of interest.

\section{References}

1. Szakács, Z.; Sánta, Z.; Lomoschitz, A.; Szántay, C., Jr. Self-induced recognition of enantiomers (SIRE) and its application in chiral NMR analysis. Trends Anal. Chem. 2018, 109, 180-197. [CrossRef]

2. Nieminen, V.; Murzin, D.Y.; Klika, K.D. NMR and molecular modeling of the dimeric self-association of the enantiomers of 1,1'bi-2-naphthol and 1-phenyl-2,2,2-trifluoroethanol in the solution state and their relevance to enantiomer self-disproportionation on achiral-phase chromatography (ESDAC). Org. Biomol. Chem. 2009, 7, 537-542. [CrossRef]

3. Baumann, A.; Wzorek, A.; Soloshonok, V.A.; Klika, K.D.; Miller, A.K. Potentially Mistaking Enantiomers for Different Compounds Due to the Self-Induced Diastereomeric Anisochronism (SIDA) Phenomenon. Symmetry 2020, 12, 1106. [CrossRef]

4. Storch, G.; Haas, M.; Trapp, O. Attracting Enantiomers: Chiral Analytes That Are Simultaneously Shift Reagents Allow Rapid Screening of Enantiomeric Ratios by NMR Spectroscopy. Chem. Eur. J. 2017, 23, 5414-5418. [CrossRef] [PubMed]

5. Klika, K.D.; Budovská, M.; Kutschy, P. Enantiodifferentiation of phytoalexin spirobrassinin derivatives using the chiral solvating agent $(R)-(+)-1,1^{\prime}$-bi-2-naphthol in conjunction with molecular modeling. Tetrahedron Asymmetry 2010, 21, 647-658. [CrossRef]

6. Klika, K.D.; Budovská, M.; Kutschy, P. NMR spectral enantioresolution of spirobrassinin and 1-methoxy-spirobrassinin enantiomers using (S)-(-)-ethyl lactate and modeling of spirobrassinin self-association for rationalization of its self-induced diastereomeric anisochronism (SIDA) and enantiomer self-disproportionation on achiral-phase chromatography (ESDAC) phenomena. J. Fluor. Chem. 2010, 131, 467-476.

7. Ouryupin, A.B.; Kadyko, M.I.; Petrovskii, P.V.; Fedin, E.I.; Okruszek, A.; Kinas, R.; Stec, W.J. Enantiomeric 2-Anilino-2-oxo1,3,2-oxazaphosphorinanes: Synthesis and NMR-Investigation of Their Non-racemic Mixtures. Tetrahedron Asymmetry 1995, 6, 1813-1824. [CrossRef]

8. Jursic, B.S.; Goldberg, S.I. Enantiomer Discrimination Arising from Solute-Solute Interactions in Partially Resolved Chloroform Solutions of Chiral Carboxamides. J. Org. Chem. 1992, 57, 7172-7174. [CrossRef]

9. Cung, M.T.; Marraud, M.; Neel, J. Experimental Study on Aggregation of Model Dipeptide Molecules. V. Stereoselective Association of Leucine Dipeptides. Biopolymers 1978, 17, 1149-1173. [CrossRef]

10. Kabachnik, M.I.; Mastryukova, T.A.; Fedin, E.I.; Vaisberg, M.S.; Morozov, L.L.; Petrovsky, P.V.; Shipov, A.E. An NMR Study of Optical Isomers in Solution. Tetrahedron 1976, 32, 1719-1728. [CrossRef]

11. Fedin, E.I.; Davankov, V.A. NMR Investigations of the Enantiomeric Excess Effects in Solutions with Weak Intermolecular Association. Chirality 1995, 7, 326-330. [CrossRef]

12. Horeau, A.; Guetté, J.P. Interactions Diastereoisomeres d'Antipodes en Phase Liquide. Tetrahedron 1974, 30, 1923-1931. [CrossRef]

13. Ouryupin, A.B.; Kadyko, M.I.; Petrovskii, P.V.; Fedin, E.I.; Sanshe, M.; Wolf, R.; Mastryukova, T.A.; Kabachnik, M.I. NMR determination of enantiomeric composition in the case of statistically controlled associate-diastereomer anisochrony. Zh. Obshch. Khim. 1995, 65, 18-22.

14. Ajisaka, K.; Kainosho, M. Diastereomeric Interaction of Partially Resolved Amines Facilitated by Lanthanide Chelates. Evidence for Dynamic Equilibrium between Seven-Coordinate and Eight-Coordinate Alkylamine-Lanthanide Chelate Adducts. J. Am. Chem. Soc. 1975, 97, 1761-1765. [CrossRef]

15. Reuben, J. Aqueous Lanthanide Shift Reagents. 8. Chiral Interactions and Stereochemical Assignments of Chemically and Isotopically Chiral Ligands. J. Am. Chem. Soc. 1980, 102, 2232-2237. [CrossRef]

16. Ouryupin, A.B.; Kadyko, M.I.; Petrovskii, P.V.; Fedin, E.I. Chiral Discrimination in Nonracemic Mixtures of Methanephosphonic Acid, N, N'-Bis(1-phenylethyl) diamides. Chirality 1994, 6, 1-4. [CrossRef] 
17. Fedin, E.I.; Morozov, L.L.; Petrovskii, P.V.; Vaisberg, M.S.; Shipov, A.E.; Mastryukova, T.A.; Kabachnik, M.I. Statistically controlled associate-diastereomeric anisochronism. Temperature dependence. Dokl. Akad. Nauk. 1974, 219, 1181-1184.

18. Kabachnik, M.I.; Mastryukova, T.A.; Shipov, A.E.; Vaisberg, M.S.; Petrovskii, P.V.; Morozov, L.L.; Fedin, E.I. Associatediastereomerism. Statistically controlled nonequivalence of the magnetic screening of nuclei in solutions of optical antipodes in achiral solvents. Dokl. Akad. Nauk. 1974, 215, 1153-1156.

19. Kabachnik, M.I.; Mastryukova, T.A.; Fedin, E.I.; Vaisberg, M.S.; Morozov, L.L.; Petrovskii, P.V.; Shipov, A.E. Optical Isomers in Solution Investigated by Nuclear Magnetic Resonance. Russ. Chem. Rev. 1978, 47, 821-834. [CrossRef]

20. Kabachnik, M.I.; Fedin, E.I.; Morozov, L.L.; Petrovskii, P.V.; Vaisberg, M.S.; Shipov, A.E.; Mastryukova, T.A. Theoretical analysis of NMR spectra of associate-diastereomers. Dokl. Akad. Nauk. 1974, 215, 1400-1403.

21. Klika, K.D. Use of sub-stoichiometric amounts of chiral auxiliaries for enantiodifferentiation by NMR; caveats and potential utility. Tetrahedron Asymmetry 2009, 20, 1099-1102. [CrossRef]

22. Soloshonok, V.A. Remarkable amplification of the self-disproportionation of enantiomers on achiral-phase chromatography columns. Angew. Chem. Int. Ed. 2006, 45, 766-769. [CrossRef] [PubMed]

23. Soloshonok, V.A.; Roussel, C.; Kitagawa, O.; Sorochinsky, A.E. Self-disproportionation of enantiomers via achiral chromatography: A warning and an extra dimension in optical purifications. Chem. Soc. Rev. 2012, 41, 4180-4188. [CrossRef]

24. Soloshonok, V.A.; Klika, K.D. Terminology related to the phenomenon 'self-disproportionation of enantiomers' (SDE). Helv. Chem. Acta 2014, 97, 1583-1589. [CrossRef]

25. Han, J.; Kitagawa, O.; Wzorek, A.; Klika, K.D.; Soloshonok, V.A. The self-disproportionation of enantiomers (SDE): A menace or an opportunity? Chem. Sci. 2018, 9, 1718-1739. [CrossRef]

26. Han, J.; Soloshonok, V.A.; Klika, K.D.; Drabowicz, J.; Wzorek, A. Chiral sulfoxides: Advances in asymmetric synthesis and problems with the accurate determination of the stereochemical outcome. Chem. Soc. Rev. 2018, 47, 1307-1350. [CrossRef]

27. Wzorek, A.; Klika, K.D.; Drabowicz, J.; Sato, A.; Aceña, J.L.; Soloshonok, V.A. The self-disproportionation of the enantiomers (SDE) of methyl $n$-pentyl sulfoxide via achiral, gravity-driven column chromatography: A case study. Org. Biomol. Chem. 2014, 12, 4738-4746. [CrossRef] [PubMed]

28. Suzuki, Y.; Han, J.; Kitagawa, O.; Aceña, J.L.; Klika, K.D.; Soloshonok, V.A. A comprehensive examination of the selfdisproportionation of enantiomers (SDE) of chiral amides via achiral, laboratory-routine, gravity-driven column chromatography. RSC Adv. 2015, 5, 2988-2993. [CrossRef]

29. Han, J.; Wzorek, A.; Kwiatkowska, M.; Soloshonok, V.A.; Klika, K.D. The self-disproportionation of enantiomers (SDE) of amino acids and their derivatives. Amino Acids 2019, 51, 865-889. [CrossRef] [PubMed]

30. Wzorek, A.; Sato, A.; Drabowicz, J.; Soloshonok, V.A.; Klika, K.D. Enantiomeric Enrichments via the Self-Disproportionation of Enantiomers (SDE) by Achiral, Gravity-Driven Column Chromatography: A Case Study Using N-(1-Phenylethyl) acetamide for Optimizing the Enantiomerically Pure Yield and Magnitude of the SDE. Helv. Chim. Acta 2015, 98, 1147-1159. [CrossRef]

31. Kwiatkowska, M.; Marcinkowska, M.; Wzorek, A.; Pajkert, R.; Han, J.; Klika, K.D.; Soloshonok, V.A.; Röschenthaler, G.-V. The self-disproportionation of enantiomers (SDE) via column chromatography of $\beta$-amino- $\alpha, \alpha$-difluorophosphonic acid derivatives. Amino Acids 2019, 51, 1377-1385. [CrossRef]

32. Davankov, V.A. Can Enantiomers Be Separated in Achiral Chromatographic Systems? Russ. J. Phys. Chem. A 2016, 90, $2119-2121$. [CrossRef]

33. Ueki, H.; Yasumoto, M.; Soloshonok, V.A. Rational application of self-disproportionation of enantiomers via sublimation-A novel methodological dimension for enantiomeric purifications. Tetrahedron Asymmetry 2010, 21, 1396-1400. [CrossRef]

34. Aceña, J.L.; Sorochinsky, A.E.; Katagiri, T.; Soloshonok, V.A. Unconventional preparation of racemic crystals of isopropyl 3,3,3-trifluoro-2-hydroxypropanoate and their unusual crystallographic structure: The ultimate preference for homochiral intermolecular interactions. Chem. Commun. 2013, 49, 373-375. [CrossRef]

35. Kurdi, R.; Táborosi, A.; Zucchi, C.; Pályi, G. Autosolvation: Architecture and Selection of Chiral Conformers in Alkylcobalt Carbonyl Molecular Clocks. Symmetry 2014, 6, 551-565. [CrossRef]

36. Wzorek, A.; Sato, A.; Drabowicz, J.; Soloshonok, V.A. Self-disproportionation of enantiomers via achiral gravity-driven column chromatography: A case study of $N$-acyl- $\alpha$-phenylethylamines. J. Chromatogr. A 2016, 1467, 270-278. [CrossRef]

37. Sorochinsky, A.E.; Aceña, J.L.; Soloshonok, V.A. Self-disproportionation of enantiomers of chiral, non-racemic fluoroorganic compounds: Role of fluorine as enabling element. Synthesis 2013, 45, 141-152. [CrossRef]

38. Soloshonok, V.A.; Berbasov, D.O. Self-Disproportionation of Enantiomers on Achiral Phase Chromatography. One More Example of Fluorine's Magic Powers. Chim. Oggi Chem. Today 2006, 24, 44-47.

39. Soloshonok, V.A.; Berbasov, D.O. Self-Disproportionation of Enantiomers of (R)-Ethyl 3-(3,5-Dinitrobenzamido)-4,4,4trifluorobutanoate on Achiral Silica Gel Stationary Phase. J. Fluor. Chem. 2006, 127, 597-603. [CrossRef]

40. Sorochinsky, A.E.; Katagiri, T.; Ono, T.; Wzorek, A.; Aceña, J.L.; Soloshonok, V.A. Optical purifications via self-disproportionation of enantiomers by achiral chromatography: Case study of a series of $\alpha-\mathrm{CF}_{3}$-containing secondary alcohols. Chirality 2013, 25, 365-368. [CrossRef] [PubMed]

41. Soloshonok, V.A.; Ueki, H.; Yasumoto, M.; Mekala, S.; Hirschi, J.S.; Singleton, D.A. Phenomenon of Optical Self-Purification of Chiral Non-Racemic Compounds. J. Am. Chem. Soc. 2007, 129, 12112-12113. [CrossRef]

42. Yasumoto, M.; Ueki, H.; Soloshonok, V.A. Self-disproportionation of enantiomers of 3,3,3-trifluorolactic acid amides via sublimation. J. Fluor. Chem. 2010, 131, 266-269. [CrossRef] 
43. Yasumoto, M.; Ueki, H.; Soloshonok, V.A. Self-disproportionation of enantiomers of $\alpha$-trifluoromethyl lactic acid amides via sublimation. J. Fluor. Chem. 2010, 131, 540-544. [CrossRef]

44. Tsuzuki, S.; Orita, H.; Ueki, H.; Soloshonok, V.A. First principle lattice energy calculations for enantiopure and racemic crystals of $\alpha$-(trifluoromethyl)lactic acid: Is self-disproportionation of enantiomers controlled by thermodynamic stability of crystals? J. Fluor. Chem. 2010, 131, 461-466. [CrossRef]

45. Albrecht, M.; Soloshonok, V.A.; Schrader, L.; Yasumoto, M.; Suhm, M.A. Chirality-dependent sublimation of $\alpha$-(trifluoromethyl)lactic acid: Relative vapor pressures of racemic, eutectic, and enantiomerically pure forms, and vibrational spectroscopy of isolated $(S, S)$ and $(S, R)$ dimers. J. Fluor. Chem. 2010, 131, 495-504. [CrossRef]

46. Yasumoto, M.; Ueki, H.; Ono, T.; Katagiri, T.; Soloshonok, V.A. Self-disproportionation of enantiomers of isopropyl 3,3,3(trifluoro)lactate via sublimation: Sublimation rates vs. enantiomeric composition. J. Fluor. Chem. 2010, 131, 535-539. [CrossRef]

47. Katagiri, T.; Takahashi, S.; Tsuboi, A.; Suzaki, M.; Uneyama, K. Discrimination of enantiomeric excess of optically active trifluorolactate by distillation: Evidence for a multi-center hydrogen bonding network in the liquid state. J. Fluor. Chem. 2010, 131, 517-520. [CrossRef]

48. Katagiri, T.; Uneyama, K. Chiral Recognition by Multicenter Single Proton Hydrogen Bonding of Trifluorolactates. Chem. Lett. 2001, 30, 1330-1331. [CrossRef]

49. Katagiri, T.; Yoda, C.; Furuhashi, K.; Ueki, K.; Kubota, T. Separation of an Enantiomorph and Its Racemate by Distillation: Strong Chiral Recognizing Ability of Trifluorolactates. Chem. Lett. 1996, 25, 115-116. [CrossRef]

50. Han, J.; Nelson, D.J.; Sorochinsky, A.E.; Soloshonok, V.A. Self-disproportionation of enantiomers via sublimation; new and truly green dimension in optical purification. Curr. Org. Synth. 2011, 8, 310-317. [CrossRef]

51. Wang, J.; Sánchez-Roselló, M.; Aceña, J.L.; del Pozo, C.; Sorochinsky, A.E.; Fustero, S.; Soloshonok, V.A.; Liu, H. Fluorine in Pharmaceutical Industry: Fluorine-Containing Drugs Introduced to the Market in the Last Decade (2001-2011). Chem. Rev. 2014, 114, 2432-2506. [CrossRef] [PubMed]

52. Zhou, Y.; Wang, J.; Gu, Z.; Wang, S.; Zhu, W.; Aceña, J.L.; Soloshonok, V.A.; Izawa, K.; Liu, H. Next Generation of FluorineContaining Pharmaceuticals, Compounds Currently in Phase II-III Clinical Trials of Major Pharmaceutical Companies: New Structural Trends and Therapeutic Areas. Chem. Rev. 2016, 116, 422-518. [CrossRef]

53. Zhu, W.; Wang, J.; Wang, S.; Gu, Z.; Aceña, J.L.; Izawa, K.; Liu, H.; Soloshonok, V.A. Recent advances in the trifluoromethylation methodology and new $\mathrm{CF}_{3}$-containing drugs. J. Fluor. Chem. 2014, 167, 37-54. [CrossRef]

54. Zhu, Y.; Han, J.; Wang, J.; Shibata, N.; Sodeoka, M.; Soloshonok, V.A.; Coelho, J.A.S.; Toste, F.D. Modern Approaches for Asymmetric Construction of Carbon-Fluorine Quaternary Stereogenic Centers: Synthetic Challenges and Pharmaceutical Needs. Chem. Rev. 2018, 118, 3887-3964. [CrossRef] [PubMed]

55. Jacques, J.; Collet, A.; Wilen, S.H. Enantiomers, Racemates, and Resolutions; J. Wiley \& Sons, Inc.: New York, NY, USA, 1981.

56. Zhang, H.Y.; Wang, X.; Ching, C.B.; Wu, J.C. Experimental optimization of enzymic kinetic resolution of racemic flurbiprofen. Biotechnol. Appl. Biochem. 2005, 42, 67-71. [PubMed]

57. Chelius, E.C.; Reutzel-Edens, S.M.; Van den Berghe Snorek, S. Crystalline of N-[4-[2-(2-amino-4,7-dihydro-4oxo-3H-pyrrolo[2,3d]pyrimidin-5-yl)ethyl]benzoyl]-L-glutamic Acid and Process Therefor. U.S. Patent 7,138,521 B2, 21 November 2006.

58. Han, J.; Takeda, R.; Sato, T.; Moriwaki, H.; Abe, H.; Izawa, K.; Soloshonok, V.A. Optical Resolution of Rimantadine. Molecules 2019, 24, 1828. [CrossRef] [PubMed]

59. Flippen, J.L.; Gilardi, R.D. ( \pm )-2-(2-Fluoro-4-biphenyl)propionic Acid (Flurbiprofen). Acta Cryst. 1975, B31, 926-928. [CrossRef]

60. Habibi-Yangjeh, A.; Pourbasheer, E.; Danandeh-Jenagharad, M. Prediction of Melting Point for Drug-like Compounds Using Principal Component-Genetic Algorithm-Artificial Neural Network. Bull. Korean Chem. Soc. 2008, 29, 833-841.

61. Swart, H.; Breytenbach, J.C.; Hadgraft, J.; du Plessis, J. Synthesis and transdermal penetration of NSAID glycoside esters. Int. J. Pharm. 2005, 301, 71-79. [CrossRef]

62. Pajula, K.; Taskinen, M.; Lehto, V.-P.; Ketolainen, J.; Korhonen, O. Predicting the Formation and Stability of Amorphous Small Molecule Binary Mixtures from Computationally Determined Flory-Huggins Interaction Parameter and Phase Diagram. Mol. Pharmaceutics 2010, 7, 795-804. [CrossRef]

63. Henck, J.-O.; Kuhnert-Brandstätter, M. Demonstration of the Terms Enantiotropy and Monotropy in Polymorphism Research Exemplified by Flurbiprofen. J. Pharm. Sci. 1999, 88, 103-108. [CrossRef] [PubMed]

64. Kawai, N.; Kato, N.; Hamada, Y.; Shioiri, T. New Methods and Reagents in Organic Synthesis. 35. A New Synthesis of Some Non-Steroidal Anti-Inflammatory Agents with the 2-Arylpropionic Acid Skeleton by the Use of Diphenyl Phosphorazidate (DPPA) as a 1,3-Dipole. Chem. Pharm. Bull. 1983, 31, 3139-3148. [CrossRef]

65. Smith, C.R.; RajanBabu, T.V. Catalytic Asymmetric Synthesis Using Feedstocks: An Enantioselective Route to 2-Arylpropionic Acids and 1-Arylethyl Amines via Hydrovinylation of Vinyl Arenes. J. Org. Chem. 2009, 74, 3066-3072. [CrossRef] [PubMed]

66. Tarasevych, A.V.; Sorochinsky, A.E.; Kukhar, V.P.; Chollet, A.; Daniellou, R.; Guillemin, J.-C. Partial Sublimation of Enantioenriched Amino Acids at Low Temperature. Is it Coming from the Formation of a Euatmotic Composition of the Gaseous Phase? J. Org. Chem. 2013, 78, 10530-10533. [CrossRef]

67. Clark, J.B.; Hastie, J.W.; Kihlborg, L.H.E.; Metselaar, R.; Thackeray, M.M. Definitions of terms relating to phase transitions of the solid state. Pure Appl. Chem. 1994, 66, 577-594. [CrossRef]

68. Gamsjäger, H.; Lorimer, J.W.; Scharlin, P.; Shaw, D.G. Glossary of terms related to solubility. Pure Appl. Chem. 2008, 80, 233-276. [CrossRef] 
69. Bellec, A.; Guillemin, J.-C. A simple explanation of the enhancement or depletion of the enantiomeric excess in the partial sublimation of enantiomerically enriched amino acids. Chem. Commun. 2010, 46, 1482-1484. [CrossRef]

70. Bellec, A.; Guillemin, J.-C. Attempts to explain the self-disproportionation observed in the partial sublimation of enantiomerically enriched carboxylic acids. J. Fluor. Chem. 2010, 131, 545-548. [CrossRef]

71. Carman, R.M.; Klika, K.D. Partially racemic compounds as brushtail possum urinary metabolites. Aust. J. Chem. 1992, 45, 651-657. [CrossRef]

72. Flynn, A.J.; Ford, A.; Maguire, A.R. Localized Partitioning of Enantiomers in Solid Samples of Sulfoxides: Importance of Sampling Method in Determination of Enantiopurity. J. Org. Chem. 2020, 85, 10216-10221. [CrossRef]

73. Doucet, H.; Fernandez, E.; Layzell, T.P.; Brown, J.M. The scope of catalytic asymmetric hydroboration/oxidation with rhodium complexes of 1,1'-(2-diarylphosphino-1-naphthyl)isoquinolines. Chem. Eur. J. 1999, 5, 1320-1330. [CrossRef]

74. Abás, S.; Arróniz, C.; Molins, E.; Escolano, C. Access to the enantiopure pyrrolobenzodiazepine (PBD) dilactam nucleus via self-disproportionation of enantiomers. Tetrahedron 2018, 74, 867-871. [CrossRef]

75. Wzorek, A.; Kamizela, A.; Sato, A.; Soloshonok, V.A. Self-disproportionation of Enantiomers (SDE) via achiral gravity-driven column chromatography of N-fluoroacyl-1-phenylethylamines. J. Fluor. Chem. 2017, 196, 37-43. [CrossRef]

76. Han, J.; Wzorek, A.; Soloshonok, V.A.; Klika, K.D. The self-disproportionation of enantiomers (SDE): The effect of scaling down, potential problems versus prospective applications, possible new occurrences, and unrealized opportunities? Electrophoresis 2019, 40, 1869-1880. [CrossRef]

77. Soloshonok, V.A.; Wzorek, A.; Klika, K.D. A question of policy: Should tests for the self-disproportionation of enantiomers (SDE) be mandatory for reports involving scalemates? Tetrahedron Asymmetry 2017, 28, 1430-1434. [CrossRef]

78. Han, J.; Remete, A.M.; Dobson, L.S.; Kiss, L.; Izawa, K.; Moriwaki, H.; Soloshonok, V.A.; O’Hagan, D. Next generation organofluorine containing blockbuster drugs. J. Fluor. Chem. 2020, 239, 109639. [CrossRef]

79. Mei, H.; Han, J.; White, S.; Graham, D.J.; Izawa, K.; Sato, T.; Fustero, S.; Meanwell, N.A.; Soloshonok, V.A. Tailor-Made Amino Acids and Fluorinated Motifs as Prominent Traits in the Modern Pharmaceuticals. Chem. Eur. J. 2020, 26, 11349-11390. [CrossRef]

80. Mei, H.; Remete, A.M.; Zou, Y.; Moriwaki, H.; Fustero, S.; Kiss, L.; Soloshonok, V.A.; Han, J. Fluorine-containing drugs approved by the FDA in 2019. Chin. Chem. Lett. 2020, 31, 2401-2413. [CrossRef]

81. Mei, H.; Han, J.; Klika, K.D.; Izawa, K.; Sato, T.; Meanwell, N.A.; Soloshonok, V.A. Applications of Fluorine-Containing Amino Acids for Drug Design. Eur. J. Med. Chem. 2020, 186, 111826. [CrossRef] [PubMed]

82. Mei, H.; Han, J.; Fustero, S.; Medio-Simon, M.; Sedgwick, D.M.; Santi, C.; Ruzziconi, R.; Soloshonok, V.A. Fluorine-Containing Drugs Approved by the FDA in 2018. Chem. Eur. J. 2019, 25, 11797-11819. [CrossRef]

83. Han, J.; Kiss, L.; Mei, H.; Remete, A.M.; Ponikvar-Svet, M.; Sedgwick, D.M.; Roman, R.; Fustero, S.; Moriwaki, H.; Soloshonok, V.A. Chemical Aspects of Human and Environmental Overload with Fluorine. Chem. Rev. 2021, in press. [CrossRef] [PubMed]

84. Tokunaga, E.; Yamamoto, T.; Ito, E.; Shibata, N. Understanding the Thalidomide Chirality in Biological Processes by the Self-disproportionation of Enantiomers. Sci. Rep. 2018, 8, 17131. [CrossRef]

85. Wu, D.H.; Chen, A.D.; Johnson, C.S., Jr. An Improved Diffusion-Ordered Spectroscopy Experiment Incorporating BipolarGradient Pulses. J. Magn. Reson. Ser. A 1995, 115, 260-264. [CrossRef]

86. McDonald, G.G.; Leigh, J.S., Jr. A New Method for Measuring Longitudinal Relaxation Times. J. Magn. Reson. 1973, 9, 358-362. [CrossRef] 